\title{
Modélisation conceptuelle globale du régime du transport particulaire sur les fleuves tropicaux d'Afrique : application aux bassins du Niger supérieur et du Bani, Mali Lumped conceptual modelling of suspended sediment transport in African tropical rivers: the Upper Niger and Bani River basins
}

\author{
C. Picouet, B. Hingray et J. C. Olivry
}

Volume 13, numéro 4, 2000

URI : https://id.erudit.org/iderudit/705403ar

DOI : https://doi.org/10.7202/705403ar

Aller au sommaire du numéro

\section{Éditeur(s)}

Université du Québec - INRS-Eau, Terre et Environnement (INRS-ETE)

ISSN

0992-7158 (imprimé)

1718-8598 (numérique)

Découvrir la revue

Citer cet article

Picouet, C., Hingray, B. \& Olivry, J. C. (2000). Modélisation conceptuelle globale du régime du transport particulaire sur les fleuves tropicaux d'Afrique : application aux bassins du Niger supérieur et du Bani, Mali. Revue des sciences de l'eau / Journal of Water Science, 13(4), 463-481.

https://doi.org/10.7202/705403ar

\section{Résumé de l'article}

Sur le fleuve Niger, les relations entre concentrations en Matières En Suspension (MES) et débits liquides montrent, à l'échelle d'une crue annuelle, des cycles d'hystérésis orthogrades. Le modèle présenté dans cet article reproduit les variations saisonnières de ces MES à partir du seul débit liquide. Il suppose que les MES proviennent de deux sources distinctes : le système " versants + réseau hydrographique secondaire ", siège d'une érosion saisonnière temporaire et le réseau hydrographique principal, siège d'une érosion permanente. Le modèle représente schématiquement la production de MES provenant de ces deux sources par le biais de deux réservoirs de MES. Le premier contient un stock en MES temporaire et limité. Ce stock, maximum au début de la crue annuelle (stock initial), est mobilisé et entraîné au cours de la saison pluvieuse en produisant un flux journalier, supposé être, à un instant donné, proportionnel au stock restant et à une fonction de puissance du débit. Le second, contient un stock de MES illimité et disponible en permanence. La mobilisation de ce stock produit un flux journalier, supposé être aussi une fonction de puissance du débit, et dont l'importance sera limité par la capacité du cours d'eau. Les cinq paramètres du modèle sont calibrés à l'aide des données acquises durant huit années hydrologiques (1991/92 à 1998/99) sur deux stations du Niger amont (Banankoro et Douna). Malgré les limites d'utilisation actuelles liées à la détermination du stock initial, le modèle présenté reconstitue de façon satisfaisante les variations annuelles des concentrations en MES et offre des perspectives intéressantes pour modéliser l'évolution temporelle des MES observées tant pour les fleuves tropicaux unimodaux que pour les petits bassins versants africains. En termes de flux annuels, le modèle n'apporte pas d'amélioration sensible par rapport à un ajustement statistique simple entre les volumes écoulés et les flux de MES. Cependant, il permet aussi de déterminer les variations de flux au cours de l'année, information qui ne peut être obtenue avec un modèle de régression statistique. 


\title{
Modélisation conceptuelle globale du régime du transport particulaire sur les fleuves tropicaux d'Afrique : application aux bassins du Niger supérieur et du Bani, Mali
}

\author{
Lumped conceptual modelling of suspended \\ sediment transport in African tropical rivers: \\ the Upper Niger and Bani River basins.
}

C. PICOUET ${ }^{\star 2}$, B. HINGRAY ${ }^{3}$, J.-C. OLIVRY ${ }^{1}$

Reçu le 21 avril 2000, accepté le 11 janvier 2001**.

\section{SUMMARY}

Estimating temporal variability of suspended sediment concentrations in a watershed is important for a number of reasons (e.g., sediment yield estimation, provision of imput data for reservoir sediment-deposition models and water quality models). Three different approaches have been adopted for modelling erosion and sediment transport: physical erosion models (WICKS and BATHURST, 1996); conceptual models (NEGEV, 1967; PINHEIRO and CAUSSADE, 1996); and empirical models (WALLING, 1977; ASSELMAN, 1977). Physical and conceptual models usually require rainfall intensity data. In African tropical river catchments, however, temporal and spatial variability of rainfall are not well known; in case of the Niger, water discharge is the only reliable hydrological parameter. This study proposes a model of temporal changes in suspended sediment concentrations using only water discharge data, thereby eliminating the need for rainfall parameters.

Daily discharge and weekly suspended sediment concentration data (from 1991/92 to 1998/99) gathered at two monitoring stations of the Upper Niger (Banankoro and Douna) (figure I) were used to study relationships between suspended sediment concentration and river discharge.

At each gauging station on the Niger, the relationship between water discharge and suspended sediment concentration during the annual flood is characterized by clockwise hysteresis (figure 3). Moreover, several other African single-annual-flood rivers - unimodal rivers - also exhibit this type of relationship (KATTAN et al., 1987; OLIVRY et al., 1988; ORANGE, 1992). This cyclicity suggests a three-stage description of sediment transport dynamics: (1) At the beginning of the rainfall season, sediments are imported by

1. Laboratoire d'Hydrologie, IRD (Institut de recherche pour le développement, France (anciennement ORSTOM), BP 5045, 34032 Montpellier cedex 1, France.

2. Blancherie $32, \mathrm{CH}-1022$ Chavannes près Renens, Switzerland.

3. IATE-HYDRAM/EPFL (École polytechnique fédérale de Lausanne), $\mathrm{CH}-1015$ Lausanne, Switzerland.

* Correspondance. E-mail : cpicouet@yahoo.fr

** Les commentaires seront reçus jusqu'au 14 septembre 2001. 
hill-slope surface runoff, re-entrainment of deposits in the channel network, and riverbed erosion. The first two sources consist of easily mobilizable material available throughout the catchment at the beginning of the hydrological year; (2) Sediment availability decreases with time as the soil becomes stabilised by vegetation during the rainy season. Erosion is consequently reduced, despite increased discharge; (3) During the period represented by the falling limb of the flood hydrograph, mobilizable material has been depleted or cannot be entrained; what suspended sediment there is originates upstream and from bank and bed erosion - permanently available sources. Non-seasonal sediment sources are grouped under the label of "continuous erosion".

We propose a lumped conceptual model of suspended-sediment concentration variations over the hydrological year. The model divides the erosion, transport and deposition processes into those acting on hill-slopes and those acting in the channel network, and assumes that both are explainable by water discharge $Q(t)$ alone. The hill-slope/channel distinction is based on the fact that suspended sediment transport in a river depends not only on transport, bank and bed-erosion capacity, but also on the amount of available material in the drainage catchment.

Sediment transport (in tons per day) for the hydrological year is thus computed as the sum of two independent daily contributions of sediment discharge. The first, $F_{m o b}(t)$, originates from a limited reservoir which is full at the beginning of the flood and available only temporarily, during the rainy season. At a given time $t$, the sediment input $F_{m o b}(t)$ is proportional to the amount remaining in the limited reservoir and to a power function of water discharge (equations 1,2 ). The second reservoir, temporally and quantitatively unlimited, injects a daily sediment discharge $F_{e c}(t)$ which is a power function of water discharge (equation 3 ); $F_{e c}(t)$ is limited only by river capacity. The final concentration is obtained from equation 4.

The five model parameters were calibrated with concentration and discharge data from hydrological years $1991 / 92$ to $1995 / 96$. The unlimited reservoir parameters were estimated using equation 6 with data taken during the decreasing stage. Initial sediment in the limited reservoir was estimated with equation 7 , using observed concentrations and concentrations derived from continuous erosion (equation 3). Two parameters related to the decrease in initial sediment loads were obtained by optimization of the mean Nash criterion between observed and calculated concentrations (figure 6 ). Some physical interpretations were ascribed to coefficients related to the limited reservoir.

Despite the limitations of assuming a single initial mobilizable reservoir, predictions of temporal sediment-concentration patterns during the annual flood were satisfactory (figure 7, table 2). The model also simulated some observed sediment concentration peaks associated with sudden water discharge variations during the rising limb of the annual flood. Best results were obtained by varying initial sediment reservoir estimates for different hydrological years (table 3) - the model could therefore be improved by highlighting parameters that determine sediment loads at the beginning of the hydrological year.

The model does not give better estimates of annual sediment yield than simple regression of annual water volume (table 4). However, it is able to reproduce the temporal variability of the sediment flux during the annual flood. The small size of the data set makes evaluation of the performance of this model difficult; for better assessment, it should applied to other data gathered on the same catchments or on other large tropical rivers. Model parameter values could also be explained by drainage basin characteristics.

Key-words: suspended sediment transport, erosion, hysteresis, lumped conceptual model, Niger River, Mali. 


\section{RÉSUMÉ}

Sur le fleuve Niger, les relations entre concentrations en Matières En Suspension (MES) et débits liquides montrent, à l'échelle d'une crue annuelle, des cycles d'hystérésis orthogrades. Le modèle présenté dans cet article reproduit les variations saisonnières de ces MES à partir du seul débit liquide. Il suppose que les MES proviennent de deux sources distinctes : le système " versants + réseau hydrographique secondaire ", siège d'une érosion saisonnière temporaire et le réseau hydrographique principal, siège d'une érosion permanente. Le modèle représente schématiquement la production de MES provenant de ces deux sources par le biais de deux réservoirs de MES. Le premier contient un stock en MES temporaire et limité. Ce stock, maximum au début de la crue annuelle (stock initial), est mobilisé et entraîné au cours de la saison pluvieuse en produisant un flux journalier, supposé être, à un instant donné, proportionnel au stock restant et à une fonction de puissance du débit. Le second, contient un stock de MES illimité et disponible en permanence. La mobilisation de ce stock produit un flux journalier, supposé être aussi une fonction de puissance du débit, et dont l'importance sera limité par la capacité du cours d'eau. Les cinq paramètres du modèle sont calibrés à l'aide des données acquises durant huit années hydrologiques (1991/92 à 1998/99) sur deux stations du Niger amont (Banankoro et Douna). Malgré les limites d'utilisation actuelles liées à la détermination du stock initial, le modèle présenté reconstitue de façon satisfaisante les variations annuelles des concentrations en MES et offre des perspectives intéressantes pour modéliser l'évolution temporelle des MES observées tant pour les fleuves tropicaux unimodaux que pour les petits bassins versants africains. En termes de flux annuels, le modèle n'apporte pas d'amélioration sensible par rapport à un ajustement statistique simple entre les volumes écoulés et les flux de MES. Cependant, il permet aussi de déterminer les variations de flux au cours de l'année, information qui ne peut être obtenue avec un modèle de régression statistique.

Mots clés : Matières en Suspension, érosion, hystérésis, modèle conceptuel, fleuve Niger, Mali.

\section{1 - INTRODUCTION}

Il est classique de chercher à corréler les concentrations des Matières En Suspension (MES) mesurées avec les débits liquides pour calculer les flux de MES transportés. Ces relations entre concentration en sédiment $C$ et débit liquide $Q$ s'avèrent plus ou moins complexes suivant les bassins étudiés mais sont généralement ajustées à des équations de la forme $C=a Q^{b}$ (avec $b>1$ ) (HASNAIN, 1996 ; JANSSON, 1996 ; FERGUSON, 1986 ; WALLING, 1977). Cette relation univoque est rarement valable pour représenter les cycles d'hystérésis observés à différentes échelles d'espace et de temps sur la plupart des bassins (ASSELMAN, 1999 ; BRUNET et GAZELLE, 1995 ; ZHANG et al., 1990 ; WALLING et WEBB, 1981). Des hystérésis de crues orthogrades à l'échelle annuelle ont par exemple été reportées pour les fleuves tropicaux unimodaux d'Afrique (une seule crue annuelle) : Niger, Cameroun, Oubangui, (OLIVRY et al., 1988 ; OLIVRY et al., 1995b), Sénégal (KATTAN et al., 1987 ; ORANGE, 1992).

Les seuls modèles qui permettent de suivre cette évolution de la charge en sédiments au cours du temps sont soit à base physique, soit conceptuels 
(NEGEV, 1967 ; PINHEIRO et CAUSSADE, 1996 ; WICKS et BATHURST, 1996). Or, la quasi-totalité des modèles existants nécessitent comme variable d'entrée la pluie et en particulier l'intensité des précipitations. Dans le cas du bassin supérieur du Niger, la taille du bassin et de ses sous-bassins ne permet pas de prendre en compte les caractéristiques relativement mal connues de la distribution spatio-temporelle des pluies et de l'érodabilité des différents sous-bassins. Cet article a donc pour objet de présenter un modèle conceptuel élaboré et testé afin de reconstituer la variation des concentrations en matières en suspension à l'exutoire des bassins étudiés à partir de la seule utilisation du débit journalier. Cette variable est relativement bien connue en une station hydrométrique donnée et intègre de plus, de nombreux phénomènes et processus singuliers à l'intérieur du bassin versant dont les effets sont en partie lissés à l'échelle du bassin. Le modèle conceptuel présenté ici permet de décrire schématiquement deux phénomènes supposés être représentatifs de la production des MES dans le transport liquide, l'érosion des versants du bassin et l'érosion des berges du cours d'eau. Dans une précédente étude (PICOUET, 1999), ce modèle conceptuel est apparu plus intéressant qu'un modèle de type empirique qui identifiait des relations entre la concentration en matières en suspension mesurée dans la rivière, le débit à l'exutoire et le volume cumulé écoulé à l'exutoire depuis le début de la crue.

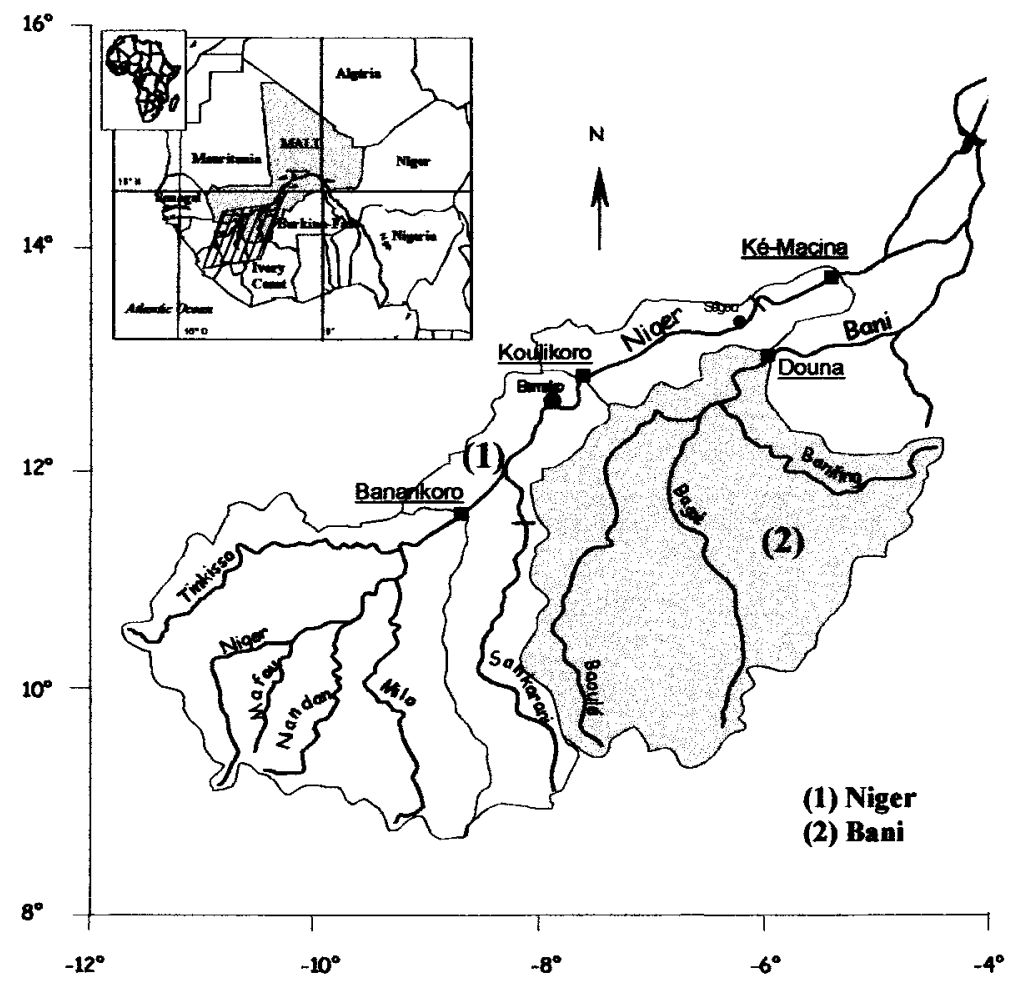

Figure 1 Situation du bassin supérieur du Niger et du Bani et stations d'échantillonnage.

Upper Niger Watershed and location of sampling sites. 


\section{2 - ZONE D'ÉTUDE : LE BASSIN SUPÉRIEUR DU NIGER}

Le bassin du Niger supérieur se limite vers l'aval à la région de Ségou et s'étend entre $8^{\circ} 35^{\prime}$ et $14^{\circ}$ de latitude Nord et entre $4^{\circ}$ et $11^{\circ} 30^{\prime}$ de longitude Ouest (figure 1). Il est essentiellement constitué d'une zone de plateaux s'inclinant progressivement vers le Nord-Est, encadré au Nord-Ouest par le plateau Mandingue qui vient longer le fleuve, à l'Ouest par le massif du Fouta-Djallon et au Sud-Ouest par les nombreuses chaînes d'origines géologiques très différentes qui viennent s'y raccorder. Le Haut-Niger reçoit sur le territoire guinéen trois affluents importants : le Tinkisso, le Niandian et le Milo. Puis parvenu au Mali, il reçoit à l'amont de Bamako, le Sankarani d'origine essentiellement guinéenne. Le Niger ne reçoit plus ensuite que des affluents mineurs jusqu'à sa confluence avec le Bani, son principal affluent, à Mopti.

Le bassin amont du Niger couvre du Sud au Nord les domaines climatiques guinéen, soudanien puis sahélien. De l'amont vers l'aval du bassin, la pluviométrie annuelle passe de $1500 \mathrm{~mm} \cdot \mathrm{an}^{-1}$ à $600 \mathrm{~mm} \cdot \mathrm{an}^{-1}$. La situation plus septentrionale du bassin du Bani explique que la pluviométrie moyenne pour la période de 1950 à 1990 n'est que de $1110 \mathrm{~mm}$ au lieu de $1450 \mathrm{~mm}$ pour le bassin du Niger à Koulikouro. Le régime des précipitations commande la grande variabilité saisonnière des débits du fleuve. Pendant six mois (janvier à juin) les débits des basses-eaux du Niger représentent moins de $7 \%$ du débit annuel. La remontée des débits s'annonce en mai, mais ne devient vraiment significative qu'au mois de juillet. Le maximum de crue a lieu généralement dans la deuxième quinzaine de septembre. La décrue est rapide et dès fin novembre, on observe la phase de vidange des réserves souterraines.

\section{3 - DESCRIPTION DES DONNÉES}

Les deux bassins versants étudiés (figure 1) se situent en amont de la station de Banankoro sur le Niger $\left(71800 \mathrm{~km}^{2}\right)$ et en amont de la station de Douna sur le Bani $\left(102000 \mathrm{~km}^{2}\right.$ ). Pour ces deux stations, le régime hydrologique peut être considéré comme naturel. Pour huit années hydrologiques complètes (de 1991/92 à 1998/99), on dispose d'observations ponctuelles sur les débits écoulés (observations journalières) et sur les concentrations en MES (observations généralement hebdomadaires). Elles ont été récoltées dans le cadre d'un programme d'étude de l'IRD sur la Géosphère Intertropicale. La description de ces données de même que la méthodologie utilisée pour l'échantillonnage, leur analyse et leur validation sont présentées dans PICOUET (1999).

II est intéressant de noter que la période d'étude regroupe des années hydroclimatiques contrastées représentatives des fluctuations hydrologiques de ces 25 dernières années (figure 2). Les débits moyens inter-annuels pour la période de 1991 à 1998 sont respectivement de 740 et $220 \mathrm{~m}^{3} \cdot \mathrm{s}^{-1}$ à Banankoro et Douna. La concentration moyenne interannuelle en MES est de $24 \mathrm{mg}^{-1}$ pour le Niger et atteint $59 \mathrm{mg} \cdot \mathrm{l}^{-1}$ pour le Bani. Les maximums de concentrations en MES, atteints en montée de crue, sont également relativement diffé- 


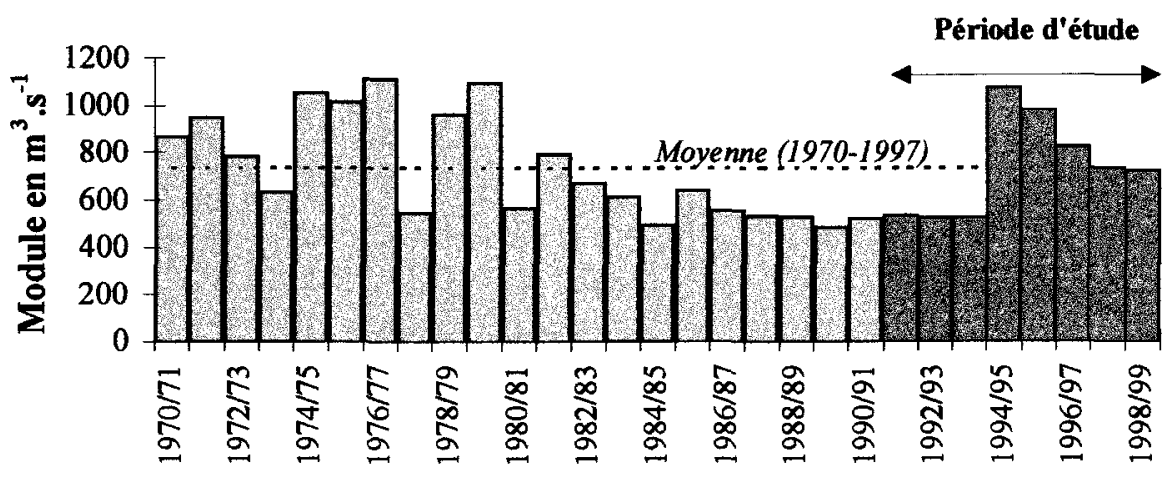

Figure 2 Écoulement annuel à la station de Banankoro (Niger supérieur). Représentativité de la période d'observation des flux particulaires par rapport aux écoulement annuel mesuré depuis 1970.

Annual runoff $\left(\mathrm{m}^{3} \cdot \mathrm{s}^{-1}\right)$ at the Banankoro gauging station (Upper Niger) from $1970 / 1971$ to $1997 / 98$.

rents entre les deux bassins : ils peuvent atteindre $80 \mathrm{mg} \cdot \mathrm{I}^{-1}$ à Banankoro et jusqu'à $250 \mathrm{mg} \cdot{ }^{-1}$ à Douna. Cette différence pourrait être reliée à la qualité des sols (sols tropicaux ferrugineux) et aux plus grandes superficies cultivées caractéristiques du bassin du Bani, qui en font une région plus sensible à l'érosion par rapport au bassin du Niger. Bien que les concentrations soient fortes sur le Bani, la faiblesse des écoulements est le principal facteur qui limite le transport de MES sur ce bassin versant.

Finalement, les données utilisées pour la calibration et la validation du modèle sont les valeurs hebdomadaires moyennes des débits et des concentrations en MES pour les huit années hydrologiques. Les débits hebdomadaires moyens ont été calculés simplement à l'aide des débits journaliers. Les concentrations hebdomadaires moyennes correspondent à la moyenne sur 7 jours des concentrations journalières pondérées par les débits journaliers. Les concentrations journalières non connues ont été obtenues par interpolation linéaire entre les concentrations journalières connues relatives aux deux jours les plus proches encadrant la date concernée.

\section{4 - ÉVOLUTION CONJOINTE DES CONCENTRATIONS ET DES DÉBITS AU COURS D'UNE CRUE ANNUELLE}

Les relations entre les concentrations en MES et les débits forment tant pour le fleuve Niger que pour son affluent le Bani des cycles d'hystérésis orthogrades (figure 3). Cette relation traduit des variations dans la disponibilité des sédiments et est classiquement attribuée à la succession de trois phases :

Une phase d'érosion initiale, qui se traduit par une augmentation simultanée des débits et des concentrations en MES. Au début de la saison des 
pluies, il existe un stock facilement mobilisable qui est distribué sur le bassin, à la fois sur les versants et dans le réseau hydrographique. Ce stock est en partie emporté par le ruissellement. Les MES ainsi produites par le bassin et/ou remises en suspension sont transportées par le fleuve et le pic de MES arrive donc très rapidement, précédant l'arrivée du maximum de crue.

Une phase d'érosion atténuée et de transport (durant laquelle la concentration en MES diminue alors que les débits augmentent toujours) se poursuit jusqu'au maximum de crue. Ce phénomène est à mettre en relation avec le processus classique d'épuisement du stock érodable sur le bassin versant et sans doute aussi avec la mise en place de la végétation qui s'est développée entre temps et qui joue donc de plus en plus un rôle de protection des sols. L'érosion est ralentie. La charge solide est aussi sans doute diluée par une contribution de plus en plus importante de l'écoulement souterrain (très pauvre en MES) par rapport à l'écoulement total.

Une phase de transport et alluvionnement, correspondant à la diminution conjointe des teneurs en MES et du débit, boucle le cycle annuel. Pendant la décrue, le stock mobilisable a disparu ou n'est plus accessible (arrêt des pluies + arrêt du ruissellement superficiel + présence de la végétation protectrice) ; l'essentiel de la charge en MES du fleuve provient alors du transport de la charge acquise plus en amont, de la mobilisation des dépôts dans le lit de la rivière et de l'érosion des berges du fleuve. Nous utiliserons par la suite l'expression " d'érosion continue " pour décrire l'ensemble des mécanismes qui contribuent à la charge en suspension observée dans le fleuve indépendamment de la saison. L'intensité de ce phénomène dépendra de la capacité du cours d'eau à mobiliser et transporter les particules solides.
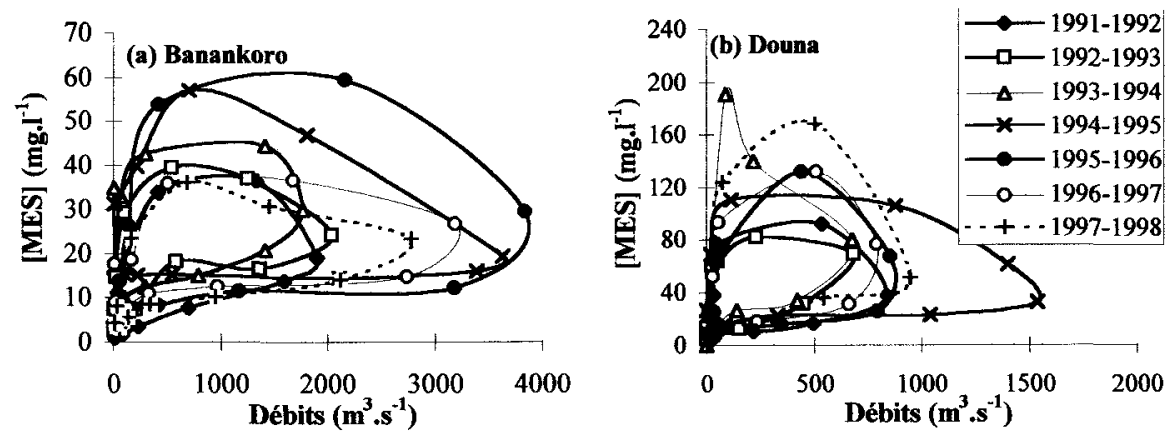

Figure 3 Relations entre les débits mensuels et les concentrations mensuelles pour chaque année et pour la station de Banankoro sur le Niger (a), et de Douna sur le Bani (b), pour la période de 1991/92 à 1998/99. La période de mai à avril représente un cycle hydrologique (une boucle).

Relationships between mean monthly water discharges and mean monthly suspended sediment concentrations at the Banankoro (a) and Douna (b) gauging stations from 1991/92 to 1998/99. The period from May to April represents one hydrological year (and one loop).

Outre la variabilité des concentrations en MES au cours de la crue annuelle, la figure 3 fait également apparaître pour une même station des variations 
importantes entre les années : de façon générale les cycles d'hystérésis relatifs aux années les plus sèches sont contenus dans les cycles relatifs aux années les plus humides. Cette variabilité interannuelle n'a pas pu être reliée aux caractéristiques hydroclimatiques générales des années étudiées (PICOUET, 1999). Elle a pu cependant être reproduite par le modèle présenté ici.

Par la suite, la totalité du débit instantané observé à une station donnée sera considérée comme pouvant expliquer la charge en sédiments transportée par le fleuve. Cette hypothèse peut sembler hasardeuse. En effet le débit transité résulte de la contribution plus ou moins différée de divers types d'écoulement (superficiels, hypodermiques, souterrains) qui n'interviennent pas forcément dans les mécanismes de l'érosion précités. Les points suivants peuvent cependant être notés :

- en début de crue, l'essentiel du débit transité résulte du ruissellement superficiel, seule composante du débit qui peut contribuer à l'érosion et au lessivage des versants. Pour cette phase, la recherche d'une relation entre le débit total observé à la station et le transport solide se justifie ;

- en phase de décrue, lorsque le débit ne provient plus que d'écoulements retardés, la période d'érosion des versants est pour ainsi dire terminée. La charge en sédiments s'explique seulement par les mécanismes relatifs à l'érosion continue dans le bassin amont. L'eau transportée dans le fleuve peut donc, quelle que soit son origine, participer à ces différents mécanismes d'érosion et le débit total transité constitue donc toujours une variable explicative pertinente de la charge instantanée en sédiments.

\section{5 - LE MODĖLE CONCEPTUEL}

\subsection{Principe de la modélisation}

Le modèle conceptuel est basé sur le fait que la quantité de matériaux en suspension dans un cours d'eau dépend d'une part de la capacité du bassin versant à alimenter le cours d'eau en particules lors des épisodes pluvieux et d'autre part de la capacité du cours d'eau à éroder les berges et le fond de lit ainsi que de sa capacité de transport amont-aval des MES.

Le principe du modèle est donc de considérer que le flux journalier (en tonnes par jour) transporté par le débit à l'exutoire $Q(t)$ est la somme d'un flux journalier $F_{m o b}(t)$ provenant d'un stock “ temporaire " et limité et d'un flux journalier $F_{e c}(t)$ provenant d'un stock permanent et illimité. Les modèles de comportement du réservoir temporaire et du réservoir permanent sont :

\subsubsection{Réservoir dont le stock est « temporaire " et " limité "}

L'hypothèse principale du modèle est qu'il existe un stock initial de matière facilement mobilisable au début de la crue $\left(M_{\text {mob }}\right.$ au temps $\left.t=0\right)$. Ce stock est entraîné au cours de la saison pluvieuse jusqu'à son épuisement plus ou moins total ; cet épuisement correspond à une diminution des particules disponibles, non renouvelées du fait de la protection progressive des sols par la végétation puis de l'arrêt de la pluie. Ce stock disponible au début de l'année hydrolo- 
gique est donc supposé ne pas pouvoir se reconstituer pendant la période pluvieuse. Son érosion produit le flux journalier de particules en suspension $F_{\text {mob }}(t)$ qui est relié à la variation du stock de sédiments disponible dans le réservoir par l'équation de continuité suivante :

$$
\frac{d M_{m o b}(t)}{d t}=-F_{m o b}(t)
$$

avec $d t$ en jours ; $M_{\text {mob }}(t)$ la quantité de sédiments à l'instant $t$, restant dans le réservoir temporaire et limité en tonnes ; $F_{\text {mob }}(t)$, le flux journalier provenant de ce stock, en tonnes par jour.

Le flux journalier $F_{m o b}(t)$ est supposé être, à un instant donné, proportionnel à deux variables : au stock restant $M_{\text {mob }}(t)$ et à une fonction puissance du débit observé à l'exutoire du bassin à cet instant.

$$
F_{\text {mob }}(t)=k_{\text {mob }} \cdot M_{\text {mab }}(t) \cdot Q(t)^{B_{m o b}}
$$

avec $Q(t)$ en $\mathrm{m}^{3} \cdot \mathrm{s}^{-1}, M_{m o b}(t)$ en $\mathrm{t}, F_{m a b}(t)$ en $\mathrm{t}$ jour ${ }^{-1}, k_{m o b}$ et $B_{m o b}$ sont des constantes.

Remarque : La relation puissance entre $F_{\text {mob }}$ et $Q$ reprend simplement la relation puissance $C=a Q^{b}$ consacrée dans la littérature scientifique entre la concentration $C$ et le débit $Q$ (voir introduction), relation également utilisable $a$ fortiori entre le flux $F$ et le débit $Q\left(F=C Q=a Q^{b+1}\right)$. Dans l'équation 2, la constante multiplicative a est en fait supposée varier dans le temps et dépendre de l'état d'un stock de MES mobilisé au fur et à mesure de la crue. ASSELMAN (1999) a proposé une approche similaire pour modéliser l'évolution des concentrations en MES au cours des crues du Rhin, la constante a étant alors supposée être une fonction exponentielle décroissante du stock progressivement mobilisé. Une relation exponentielle introduit cependant un paramètre supplémentaire à calibrer. La relation linéaire à débit constant entre $F_{m o b}(t)$ et $M_{m o b}(t)$ utilisée ici (équation 2) fournit des résultats très satisfaisants et a donc été retenue.

\subsubsection{Réservoir dont le stock est « permanent » et « illimité "}

La mobilisation de ce stock n'est limitée que par la capacité du cours d'eau à mobiliser et transporter les particules solides. II correspond à l'érosion dite continue définie auparavant. Le flux journalier provenant de l'érosion continue $\left(F_{e c}(t)\right)$ est supposé être aussi une fonction puissance du débit (la remarque effectuée pour la relation (équation 2) s'applique ici aussi) :

$$
F_{\mathrm{ec}}(t)=k_{\mathrm{ec}} \cdot \mathrm{Q}(t)^{B_{\mathrm{ec}}}
$$

avec $Q(t)$ en $\mathrm{m}^{3} \mathrm{~s}^{-1}$, et $F_{e c}(t)$ en $t$ jour ${ }^{-1} . k_{e c}$ et $B_{e c}$ sont des constantes.

À partir de ces deux flux $\left(F_{\text {mob }}(t)\right.$ et $\left.F_{e c}(t)\right)$, la concentration hebdomadaire en MES $\left(C_{c a l c}\right)$ à l'instant $t$ est finalement donnée par :

$$
C_{c a l c}(t)=\frac{1}{A}\left[\left(\frac{F_{m o b}(t)}{Q(t)}\right)+\left(\frac{F_{e c}(t)}{Q(t)}\right)\right] \text { i.e. } C_{c a l c}(t)=C_{m o b}+C_{e c}
$$

avec $F_{\text {ec }}(t)$ et $F_{\text {mob }}(t)$ en $\mathrm{t}$ jour ${ }^{-1}, Q(t)$ en $\mathrm{m}^{3} \mathrm{~s}^{-1}, C_{\text {calc }}(t)$ en $\mathrm{mg}^{-1}$ et où $A$ est une constante permettant le changement d'unités entre ces différentes variables. 


\subsection{Influence des paramètres sur la courbe de concentration calculée}

Le rôle des paramètres relatifs à l'érosion continue est simple (équation 3). Le paramètre $k_{e c}$ est la concentration obtenue du fait de l'érosion continue lorsque le débit écoulé est de $1 \mathrm{~m}^{3} \cdot \mathrm{s}^{-1}$. L'exposant $B_{e c}$ règle la plus ou moins grande sensibilité des variations de la concentration correspondant à l'érosion continue $C_{e c}(t)$ aux variations de $Q(t)$. En effet, d'après les équations (équations 3 et 4$), \Delta C_{e c}(t) / C_{e c}(t)=\left(B_{e c}-1\right) \Delta Q(t) / Q(t)$; ainsi, si $B_{e c}=1$, l'érosion continue contribue à une concentration constante indépendante du débit de valeur $k_{e c} ;$ si $B_{e c}=2, C_{e c}(t)$ varie proportionnellement à $Q(t)$, etc.

Le rôle des autres paramètres relatifs à l'érosion du stock mobilisable $M_{\text {mob }}(t)$, est plus complexe. L'équation 2 permet de déduire qu'à un instant donné et pour un débit $Q(t)$ donné, le flux provenant du stock mobilisable $F_{\text {mob }}(\mathrm{t})$ sera d'autant plus grand que $M_{\text {mob }}(t), k_{\text {mob }}$ et $B_{\text {mob }}$ seront grands et par conséquent la concentration $C_{m o b}(t)$ sera d'autant plus importante. De plus, pour un hydrogramme de crue $Q(t)$ donné, l'érosion relative du stock mobilisable $\left(d M_{m o b}(t) / M_{m o b}(t)\right)$ sera d'autant plus forte et par suite l'érosion du stock mobilisable d'autant plus rapide que $k_{m o b}$ et $B_{m o b}$ seront grands. L'influence du débit et des variations de débit sur les variations de concentration sont d'autant plus appréciables que le paramètre $B_{\text {mob }}$ est grand. En considérant l'expression de la dérivée temporelle de $C_{\text {mob }}(t)$ (équation 5 ), on déduit :

$$
\begin{aligned}
& \frac{d C_{m a b}(t)}{d t}=k_{m a b}^{2} \cdot M_{m o b}(t) \cdot Q(t)^{2 B_{m a b}-2} S(t) \\
& S(t)=\left[\frac{\left(B_{m a b}-1\right)}{k_{m o b}} \cdot \frac{1}{Q(t)^{B_{m o b}}} \cdot \frac{d Q(t)}{d t}-1\right]
\end{aligned}
$$

(1) Si $B_{m o b} \leq 1$, alors la concentration $C_{m o b}(t)$ est une fonction strictement décroissante du temps écoulé depuis le début de la crue tant que le débit augmente (signe négatif de $S(t)$ dans équation 5 ). La concentration $C_{\text {mob }}(t)$ maximum est alors observée au commencement de la saison des pluies. Le calage du modèle pour de telles valeurs de $B_{\text {mob }}$ est par suite impossible ; (2) : Si $B_{\text {mob }}$ $>1$, alors la concentration $C_{\text {mob }}(t)$ est une fonction qui peut être alternativement croissante ou décroissante (selon le signe de $S(t)$ ).

\subsection{Estimation des paramètres du modèle}

Les cinq premières années d'étude (1991/92 à 1995/96) sont utilisées pour la calibration du modèle. Cinq paramètres, $k_{m o b}, M_{m o b}(t=0), B_{m o b}, k_{e c}$ et $B_{e c}$ doivent être déterminés.

Les paramètres $k_{e c}$ et $B_{e c}$ sont obtenus à partir des données $\left\langle Q_{j}(t), C_{j}(t)\right)$ relatives à la phase de décrue pendant laquelle seule la contribution due à l'érosion continue entre en compte (i.e., $F_{\text {mob }}(t)$ est nul). La concentration hebdomadaire $C_{\text {calc }}(t)$ peut alors s'exprimer simplement de la façon suivante:

$$
C_{c a i c}(t)=C_{e c}(t)=\frac{k_{e c}}{A} \cdot Q(t)^{B_{e c}-1}
$$

Les paramètres $k_{e c}$ et $B_{e c}$ correspondent aux coefficients de l'ajustement logarithmique simple entre les concentrations et les débits moyens hebdoma- 


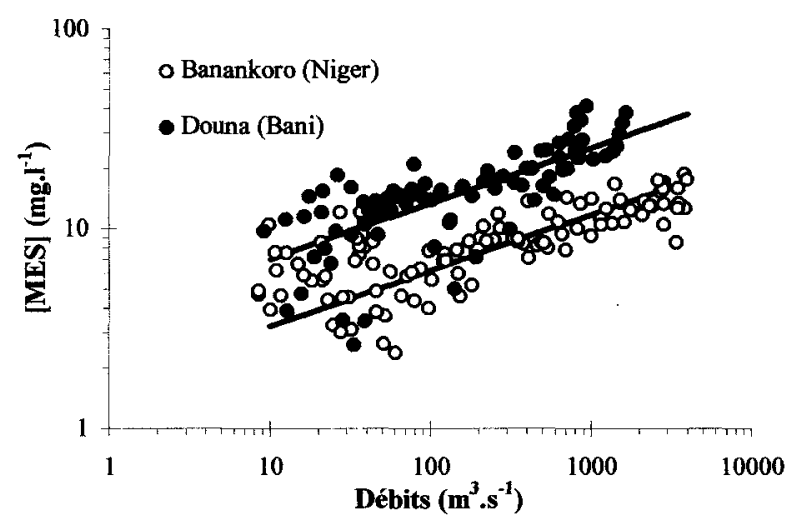

Figure 4 Relation entre la concentration et le débit hebdomadaire en période de récession de crue et de basses eaux pour les années de forte hydraulicité (pour les deux stations de Banankoro et Douna). Cette relation est utilisée pour détermination les paramètres kec et Bec relatifs au flux de MES provenant de l'érosion continue (Fec (t)).

Correlation between water discharge and suspended sediment concentration during the recession limb. The model uses this relation to estimate parameters kec and Bec for the calculation of daily sediment discharge originating from continuous erosion (Fec $(t))$.

daires observés pendant la période de décrue (figure 4). Une méthode d'ajustement graphique a été préférée à une méthode d'ajustement par les moindres carrés dans le but de donner moins d'importance aux concentrations observées pour les faibles débits. Celles-ci présentent en effet une forte variation qui est rarement en relation avec les variations de débits (faible tranche d'eau soumise aux perturbations telles que vent, passage à guet...). Différents jeux ( $k_{e c}$, $B_{e c}$ ) permettant de retrouver des résultats d'une qualité équivalente, nous avons choisi arbitrairement d'utiliser un même exposant $B_{\mathrm{ec}}$ pour les deux stations (et donc des droites de même pente dans le graphique log-log).

Ensuite, le stock facilement mobilisable en début de crue, $M_{\text {mob }}(0)$, peut pour chaque crue être évalué en calculant l'intégrale suivante (figure 5) :

$$
M_{\text {mob }}(0)=A \int_{\text {Année }}\left(C_{\text {obs }}(t)-C_{\text {ec }}(t)\right) \cdot Q(t) \cdot d t
$$

L'érosion de ce stock $M_{m o b}(0)$ explique le surplus de concentration observé dans l'écoulement en début de crue par rapport à la concentration en MES provenant uniquement de l'érosion continue. La figure $5 a$ et $5 b$ présente, pour la station de Douna, la méthode permettant de déterminer le stock $M_{\text {mob }}(0)$ pour l'année 1991-1992. Pour une station donnée, la valeur de ce paramètre varie d'une année hydrologique à une autre mais il n'a pas été possible jusqu'à présent d'identifier les variables explicatives de cette variabilité. La solution retenue afin de déterminer le stock initial $M_{m o b}(0)$ pour les années hydrologiques servant à la validation du modèle est donc d'utiliser un stock initial unique pour toutes les années. Celui-ci correspond à la moyenne des stocks initiaux obtenus grâce à l'équation (équation 7) pour les cinq années utilisées pour le calage du modèle. 
(a)

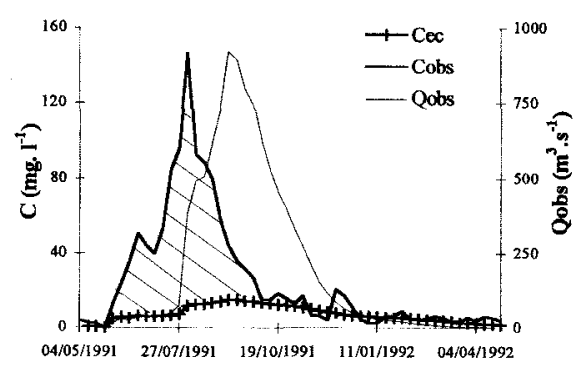

(b)

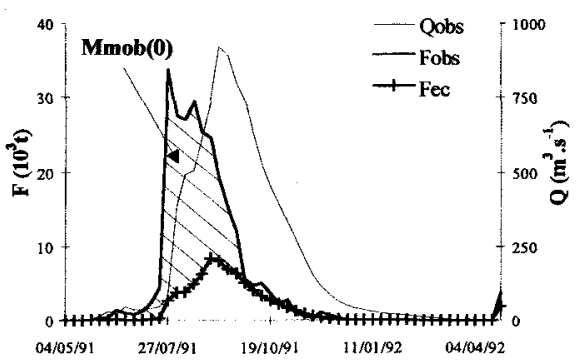

Figure 5 Détermination du stock mobilisable initial pour l'année 1991-1992 à Douna en calculant l'intégrale de l'équation (équation 7), i.e. l'aire de la surface comprise entre la courbe des flux hebdomadaires observés et la courbe des flux hebdomadaires provenant de l'érosion continue. (a) concentrations hebdomadaires observées et contribution due à l'érosion continue $\left(\mathrm{C}_{\mathrm{ec}}(\mathrm{t})\right)$. (b) flux hebdomadaires observés $\left(\mathrm{F}_{\mathrm{obs}}(\mathrm{t})\right)$ et contribution due à l'érosion continue $\left(F_{e c}(t)\right)$.

Calculation of the initial mobilizable reservoir $M_{\text {mob }}(0)$ for Douna gauging station (1991-1992) using the integration of equation (7), i.e., the area between the observed sediment load curve and the calculated sediment load curve originating from continuous erosion. (a)Observed suspended sediment concentration $\left(\mathrm{C}_{\text {obs }}\right.$ ) and contribution from continuous erosion $\left(C_{e c}(t)\right)$. (b) Observed sediment load $\left(F_{\text {obs }}(t)\right)$ and contribution from continuous erosion $\left(F_{\text {ec }}(t)\right)$.
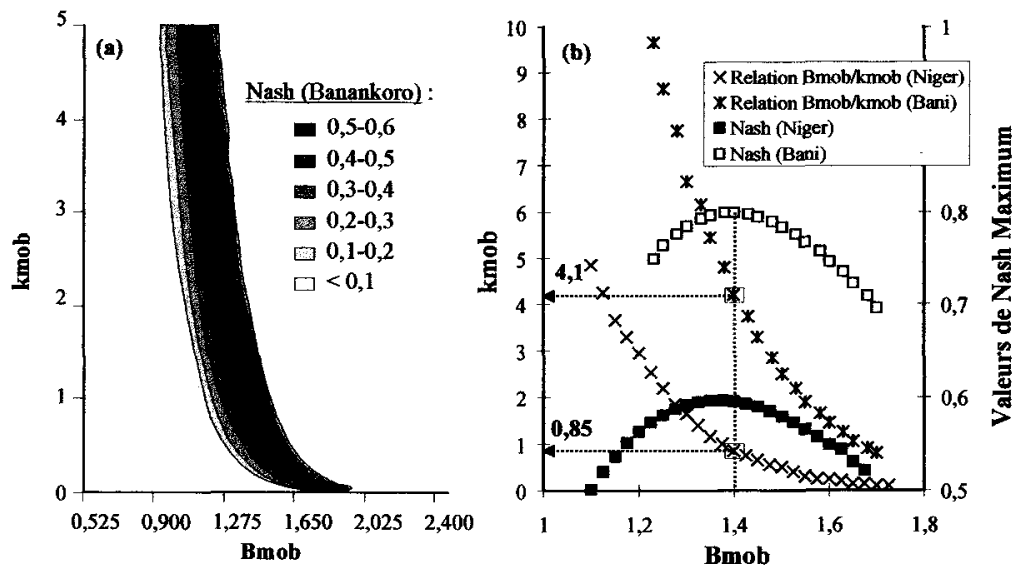

Figure 6 Détermination des paramètres $B_{m o b} k_{m o b}$ de l'équation 2. (a) Analyse de sensibilité du critère de NASH moyen aux variations des paramètres $B_{m o b}$ et $k_{m o b}$ pour la station de Banankoro (Niger). (b) Valeurs du critère de NASH maximum pour les différentes combinaisons possibles du couple $\left(B_{m o b}, k_{\text {mob }}\right)$ pour les stations de Banankoro (Niger) et Douna (Bani).

Derivation of parameters $B_{\text {mob }}$ and $k_{\text {mob }}$ (equation 2). (a) NASH criteria variability for $B_{m o b}$ and $k_{m o b}$ variability. (b) NASH criteria maxima for $B_{m o b}$ and $k_{m o b}$. 
Les deux paramètres $k_{m o b}$ et $B_{m o b}$ ne peuvent pas être prédéterminés d'une façon similaire à celle exposée ci-dessus. Ils ont tous deux été évalués de façon à obtenir la plus haute valeur possible du critère de Nash moyen (NASH et SUTCLIFFE, 1970) calculé entre l'ensemble des concentrations observées et les concentrations simulées. L'analyse de sensibilité du critère de Nash montre d'une part qu'il est plus sensible au paramètre $B_{\text {mob }}$ qu'au paramètre $k_{\text {mob }}$ et d'autre part qu'il existe une relative dépendance entre ces paramètres (figure 6a). Les valeurs du critère de NASH pour les différentes combinaisons possibles du couple $\left(B_{m o b}, k_{m o b}\right)$ permettent néanmoins d'identifier un couple de paramètres optimaux unique pour chacune des deux stations de Banankoro sur le Niger et de Douna sur le Bani (figure 6b). Les valeurs optimales du paramètre $B_{m o b}$ sont quasiment identiques pour les deux stations (un exposant $B_{\text {mob }}$ unique a d'ailleurs été utilisé par la suite) contrairement aux valeurs optimales du paramètre $k_{\text {mob }}$ très différentes entre les deux stations.

Les valeurs des paramètres optimaux sont présentées dans le tableau 1 pour chacune des deux stations étudiées.

Tableau 1 Valeurs des paramètres des différents ajustements du modèle conceptuel (les débits, les concentrations et les volumes écoulés sont respectivement exprimés en $\mathrm{m}^{3} \cdot \mathrm{s}^{-1}, \mathrm{mg} \cdot \mathrm{l}^{-1}$ et en $10^{9} \cdot \mathrm{m}^{3}$ ).

Table 1 Parameter values of the conceptual model. Mean weekly water discharge, mean weekly suspended sediment concentration and mean weekly water flow used in the conceptual model are in $\mathrm{m}^{3} \cdot \mathrm{s}^{-1}$, $m g \cdot \Gamma^{1}$ and $10^{9} \mathrm{~m}^{3}$, respectively.

\begin{tabular}{|c|c|c|c|c|c|}
\hline Station & $\boldsymbol{k}_{\boldsymbol{e c}}$ & $\boldsymbol{B}_{\boldsymbol{e c}}$ & $\boldsymbol{k}_{\boldsymbol{m a b}}$ & $\boldsymbol{B}_{\text {mob }}$ & $\boldsymbol{M}_{\boldsymbol{m o b}}(\boldsymbol{0})$ (tonnes) \\
\hline Banankoro & 0,147 & 1,28 & $0,8510^{-6}$ & 1,4 & 264000 \\
Douna & 0,319 & 1,28 & $4,110^{-6}$ & 1,4 & 232000 \\
\hline
\end{tabular}

\section{6 - RÉSULTAT ET DISCUSSION}

\subsection{Qualité des résultats}

Le modèle proposé permet de représenter l'évolution générale des concentrations au cours d'une année hydrologique pour l'ensemble des années et pour les deux stations : le maximum de concentration est systématiquement observé au milieu de la montée de crue ; la forte décroissance des concentrations qui suit, est aussi relativement bien reproduite (figure 7). Le modèle permet également de simuler certains pics de concentration observés en réponse aux variations brutales de débits lors de la montée de la crue annuelle. Seule l'intensité du pic de concentration annuel maximum n'est pas aussi bien prédite par le modèle pour toutes les années étudiées (figure 7). II est également logique que les pics secondaires observés en basses eaux ne soient pas simulés par le modèle puisqu'ils ne correspondent pas à des variations de débits mais à des phénomènes locaux déjà évoqués auparavant. La qualité de la 

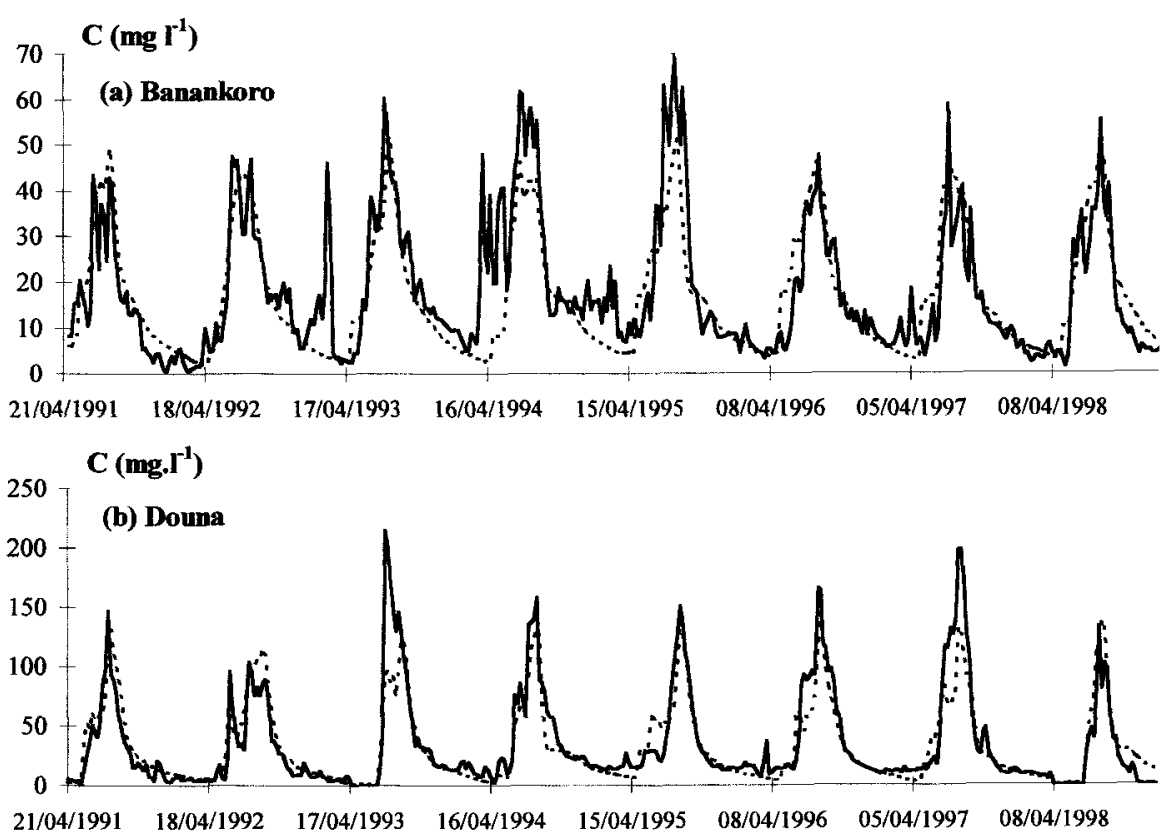

Figure 7 Comparaison de l'évolution au cours du temps des concentrations observées (ligne continue) et calculées (ligne pointillée) avec le modèle conceptuel sur les deux stations étudiées, station de Banankoro (a) et Douna (b).

Comparison of measured (continuous line) and simulated (dashed line) suspended sediment concentrations at the Banankoro (a) and Douna (b) gauging stations.

reconstitution a aussi été évaluée à l'aide du critère de Nash calculé entre concentrations hebdomadaires observées et concentrations hebdomadaires calculées. Si la qualité des résultats est assez variable suivant la station (résultats meilleurs à Douna) et suivant les années observées (tableau 2), elle reste toutefois toujours largement acceptable avec des critères de NASH moyens sur l'ensemble des années simulées de 0,63 et 0,79 respectivement à Banankoro et Douna.

\subsection{Interprétation de la valeur de différents paramètres}

Dans ce genre de modèles, considérés comme des modèles de type « boîte noire " en opposition aux modèles à bases physiques, les paramètres optimisés n'ont pas de signification physique puisqu'il englobe les effets de différents processus et incertitudes. Cependant, il est intéressant de commenter les valeurs très différentes, observées entre stations, pour les paramètres du modèle conceptuel global présenté ici (tableau 1). Le paramètre $k_{\text {mob }}$ par exemple, relatif à la rapidité d'érosion du stock initial mobilisable, est inférieur à $10^{-6}$ sur le Niger et atteint $4,1 \cdot 10^{-6}$ à Douna. La plus forte valeur observée pour le Bani témoigne d'une mobilisation plus rapide du stock temporaire et explique que les concentrations observées soient plus importantes et qu'elles 
Tableau 2 Qualité des résultats (critère de Nash calculé entre $C_{c a / c}$ et $C_{o b s}$ ) obtenus avec le modèle conceptuel pour les deux stations situées sur le bassin du Niger Amont et du Bani pour les années de calibration du modèle (1991-1992 à 1995-1996) et les années de validation du modèle (1996-1997 à 1998-1999).

Table 2 Efficiency (Nash criterion values for $C_{\text {calc }}$ and $C_{o b s}$ ) of the conceptual model during calibration years (1991-92 to 1995-96) and validation years (1996-1997 to 1998-1999).

\begin{tabular}{|c|c|c|c|c|c|c|c|c|}
\hline Slation & $\mathbf{1 9 9 1 /}$ & $\mathbf{1 9 9 2 /}$ & $\mathbf{1 9 9 3 /}$ & $\mathbf{1 9 9 4 /}$ & $\mathbf{1 9 9 5 /}$ & $\mathbf{1 9 9 6 /}$ & $\mathbf{1 9 9 7 /}$ & $\mathbf{1 9 9 8 /}$ \\
& $\mathbf{1 9 9 2}$ & $\mathbf{1 9 9 3}$ & $\mathbf{1 9 9 4}$ & $\mathbf{1 9 9 5}$ & $\mathbf{1 9 9 6}$ & $\mathbf{1 9 9 7}$ & $\mathbf{1 9 9 8}$ & $\mathbf{1 9 9 9}$ \\
\hline Banankoro & 0,69 & 0,51 & 0,41 & 0,54 & 0,77 & 0,66 & 0,69 & 0,79 \\
Douna & 0,80 & 0,78 & 0,70 & 0,82 & 0,84 & 0,84 & 0,76 & 0,75 \\
\hline
\end{tabular}

arrivent plus tôt au cours de la crue. D'après l'interprétation du paramètre a de l'équation a $Q^{b}$ (MORGAN, 1995), le paramètre $k_{\text {mob }}$ pourrait donc être caractéristique de l'érodibilité des sols, différente a priori entre les deux bassins étudiés.

Le stock initial mobilisable moyen $\left(M_{\text {mob }}(t=0)\right)$ est presque le même aux stations de Douna et de Banankoro pour un bassin de superficie plus importante sur le Bani. Le stock spécifique moyen (stock ramené à la surface) est ainsi légèrement plus faible sur le Bani. Par ailleurs, pour chaque station, le stock mobilisable initial utilisé correspond à un stock mobilisable moyen déterminé pour l'ensemble des années de calage. Pourtant de meilleurs résultats ont été trouvés pour 14 des 16 années étudiées en utilisant les valeurs de $M_{\text {mob }}(t=0)$ adaptées pour chaque année considérée (tableau 3 ). Ceci est particulièrement remarquable pour l'année 1996-1997 à la station de Banankoro. Les maximums de concentrations observés lors de la montée de crue sont de même mieux prédits.

Tableau 3 Qualité des résultats (critère de NASH) obtenus avec le modèle conceptuel amélioré (avec une valeur de $M_{\text {mob }}(t=0)$ variable).

Table 3 Efficiency (NASH criterion values) of the improved conceptual model (with $M_{\text {mob }}(t=0)$ ).

\begin{tabular}{|c|c|c|c|c|c|c|c|c|}
\hline Station & $\begin{array}{c}\mathbf{1 9 9 1 /} \\
\mathbf{1 9 9 2}\end{array}$ & $\begin{array}{c}\mathbf{1 9 9 2 /} \\
\mathbf{1 9 9 3}\end{array}$ & $\begin{array}{c}\mathbf{1 9 9 3 /} \\
\mathbf{1 9 9 4}\end{array}$ & $\begin{array}{c}\mathbf{1 9 9 4 /} \\
\mathbf{1 9 9 5}\end{array}$ & $\begin{array}{c}\mathbf{1 9 9 5 /} \\
\mathbf{1 9 9 6}\end{array}$ & $\begin{array}{c}\mathbf{1 9 9 6 /} \\
\mathbf{1 9 9 7}\end{array}$ & $\begin{array}{c}\mathbf{1 9 9 7 /} \\
\mathbf{1 9 9 8}\end{array}$ & $\begin{array}{c}\mathbf{1 9 9 8 /} \\
\mathbf{1 9 9 9}\end{array}$ \\
\hline Banankoro & 0,72 & 0,50 & 0,41 & 0,60 & 0,81 & 0,78 & 0,79 & 0,82 \\
Douna & 0,80 & 0,81 & 0,71 & 0,68 & 0,84 & 0,90 & 0,88 & 0,84 \\
\hline
\end{tabular}

Dans un but prédictif, il serait intéressant pour la modélisation conceptuelle d'expliquer, à partir de certaines caractéristiques physiques du milieu, la valeur de ce stock mobilisable $M_{\text {mob }}(t=0)$. La quantité de sédiments au début de l'année hydrologique pourrait, par exemple, être reliée à la durée de la saison sèche précédente (qui a conduit a une accumulation de poussières sur les versants d'autant plus importante que cette durée est longue) ou à l'hydrologie 
des années passées. Pour les bassins étudiés, les tentatives d'explication en fonction de l'hydraulicité de l'année précédente ont été infructueuses. Le nombre d'années et d'informations disponibles est en fait insuffisant pour poursuivre ce type d'investigations.

\subsection{Flux annuel de matières particulaires}

Lors d'une étude précédente, les flux annuels ont été calculés par la somme des flux journaliers obtenus après interpolation linéaire des concentrations hebdomadaires observées (PICOUET, 1999). Pour la reconstitution de ces flux annuels dits observés, deux méthodes sont envisagées et comparées : (1) les flux instantanés sont estimés à partir des débits et des concentrations instantanées calculées avec le modèle conceptuel. Les flux annuels en sont ensuite déduits ; (2) Les flux annuels sont directement estimés à partir des débits annuels à l'aide d'un ajustement linéaire simple $\left(F_{a n}=a Q_{a n}\right)$ effectué entre débit annuel $\left(Q_{a n}\right)$ et flux annuel $\left(F_{a n}\right)$ (ajustement réalisé sur les 5 années ayant servi au calage du modèle conceptuel). Pour chacune des crues disponibles, les écarts entre les flux annuels observés et les flux annuels calculés sont présentés dans le tableau 4 . Ces résultats montrent que : (1) l'écart entre flux observés et calculés (en \%) est très variable d'une année à une autre, quels que soient la station et le modèle utilisé ; (2) L'ajustement statistique simple donne des résultats meilleurs ou au moins équivalents à ceux observés avec le modèle conceptuel. En termes de flux annuels, le modèle conceptuel présenté dans cet article n'apporte donc pas d'amélioration sensible par rapport à l'ajustement statistique simple entre les volumes écoulés et les flux de MES. Ceci peut être relié au fait que le transport annuel dépend très peu des fortes concentrations atteintes lors de la phase d'érosion initiale au début de la montée des eaux : cette période ne pesant en moyenne que pour $12 \%$ dans le flux annuel. Le transport particulaire annuel est donc avant tout expliqué par la quantité d'eau écoulée.

Tableau 4 Écarts entre les flux annuels observés (somme des flux journaliers obtenus après interpolation linéaire des concentrations hebdomadaires) et les flux annuels calculés (résultats issus de l'ajustement statistique simple et du modèle conceptuel).

Table 4 Difference between the observed annual yield (obtained by concentration interpolation) and annual yields calculated by simple regression of annual water volume and by the lumped conceptual model.

\begin{tabular}{|c|c|c|r|r|r|r|r|r|}
\hline Station & Modèle utilisé & $\begin{array}{c}1991 / \\
1992\end{array}$ & $\begin{array}{r}1992 / \\
1993\end{array}$ & $\begin{array}{r}1993 / \\
1994\end{array}$ & $\begin{array}{r}1994 / \\
1995\end{array}$ & $\begin{array}{r}1995 / \\
1996\end{array}$ & $\begin{array}{c}1996 / \\
1997\end{array}$ & $\begin{array}{c}1997 / \\
1998\end{array}$ \\
\hline \multirow{2}{*}{ Banankoro } & Ajustement simple & $23 \%$ & $-1 \%$ & $-13 \%$ & $5 \%$ & $-11 \%$ & $8 \%$ & $20 \%$ \\
& Modèle conceptuel & $38 \%$ & $10 \%$ & $-3 \%$ & $-4 \%$ & $-16 \%$ & $7 \%$ & $20 \%$ \\
\cline { 2 - 5 } Douna & Ajustement simple & $24 \%$ & $2 \%$ & $-28 \%$ & $5 \%$ & $-4 \%$ & $-21 \%$ & $-24 \%$ \\
& Modèle conceptuel & $38 \%$ & $32 \%$ & $-6 \%$ & $-16 \%$ & $-1 \%$ & $-14 \%$ & $-18 \%$ \\
\hline
\end{tabular}




\section{7 - CONCLUSIONS}

Quelle que soit l'année d'observation, l'évolution de la concentration mensuelle en matière en suspension dans les eaux du Niger à Banankoro et du Bani à Douna en fonction du débit mensuel du fleuve montre une relation d'hystérésis orthograde classique pour les fleuves tropicaux à crue unique. Cette relation traduit principalement la diminution de la disponibilité en sédiments au cours de la saison des pluies. La relation fréquemment utilisée $C$ $=a Q^{b}$ (avec $b>1$ ) liant la concentration en MES du cours d'eau avec son débit liquide n'est donc pas adaptée pour décrire les relations MES/débits non univoques rencontrées sur les stations du bassin du Niger amont. Dans cet article, un modèle conceptuel utilisant comme variable d'entrée les seuls débits liquides a ainsi été proposé ; il prend en compte deux réservoirs de sédiments. Appliqué aux données expérimentales mesurées à la station de Banankoro sur le Niger et à la station de Douna sur le Bani pour les années hydrologiques de 1991-1992 à 1998-1999, le modèle a permis de représenter l'évolution des concentrations de MES dans le temps et également certains pics de concentrations observés lors des variations brutales de débit en début de crue. Dans l'estimation des flux solides annuels, le modèle conceptuel n'apporte pas ici un gain significatif par rapport aux estimations obtenues par un simple ajustement statistique avec les flux hydriques annuels, mais il permet cependant de déterminer les variations de flux au cours de l'année, information qui ne peut être obtenue avec le modèle de régression statistique simple.

Le modèle conceptuel, malgré ses relativement bons résultats, souffre actuellement de diverses limites. Tout d'abord, l'hypothèse que le stock mobilisable initial est identique pour toutes les années hydrologiques d'une même station est évidemment simplificatrice. Elle a cependant permis de reconstituer avec une qualité tout à fait honorable l'évolution temporelle des flux sur les années de validation et peut donc être utilisée en première approximation si l'on souhaite prédire les flux exportés pour les années futures. De meilleurs résultats sont obtenus dès lors que l'on utilise pour chaque année hydrologique un stock initial adapté. II serait donc intéressant d'étudier la façon dont se reconstitue à l'échelle du bassin versant le stock mobilisable entre deux crues annuelles pour tenter ensuite de la modéliser (par exemple modéliser une accumulation de poussières sur les versants pendant la saison sèche à la façon des modèles en hydrologie urbaine).

II faut également garder à l'esprit que les deux réservoirs de matières utilisés dans la modélisation ne reflètent pas forcément les seuls processus qui y sont attachés et que ce concept de réservoirs n'est qu'une schématisation imparfaite de la réalité. Entre le stock mobilisable des versants supposé fini à l'échelle de la saison et le réservoir de sédiments toujours disponible dans le réseau hydrographique (berges et lit), il y a toutes les contributions des drains primaires qui participent à l'alimentation des deux réservoirs. Enfin, il faut admettre que ce modèle conceptuel a intégré, sans l'identifier, l'effet de dilution de la charge solide, dilution liée à la part de plus en plus grande prise par les écoulements lents (sans charge particulaire initiale) dans l'hydrogramme de crue. Ce phénomène pourrait également expliquer, en partie, les différences entre les deux bassins étudiés. 
II est certain que la durée du jeu de données actuellement disponible est insuffisante pour réellement évaluer la performance du modèle conceptuel présenté dans cet article. II offre néanmoins des perpectives intéressantes et son potentiel mériterait d'être confirmé par l'exploitation de données supplémentaires sur les mêmes bassins et/ou sur d'autres bassins tropicaux africains. II semble notamment envisageable, en étudiant un nombre suffisant de bassins, d'expliquer la valeur de certains paramètres du modèle à partir des caractéristiques physiques du milieu et/ou des caractéristiques hydroclimatiques de la région concernée.

\section{RÉFÉRENCES BIBLIOGRAPHIQUES}

ASSELMAN N.E.M., 1999. Suspended sediment dynamics in a large drainage basin: the River Rhine. Hydrol. Process., 13, 1437-1450.

BRUNET R.C., GAZELLE F., 1995. Alternance des phénomènes d'érosion et de rétention de la matière dans la zone inondable de l'Adour au cours d'une saison hydrologique. Acta Oecologica, 16, 331-349.

FERGUSON R.I., 1986. River loads underestimated by rating curves. Water Resour. Res., 22, 74-76.

HASNAIN S.I., 1996. Factors controlling suspended sediment transport in Himalayan glacier meltwaters. J. Hydrol., 181, 4962.

JANSSON M.B., 1996. Estimating a sediment rating curve of the Reventazon river at Palomo using logged mean loads within discharge classes. J. Hydrol., 183, 227-241.

KATTAN Z., GAC J.Y., PROBST J.L., 1987. Suspended load and mechanical erosion in Senegal basin - Estimation of the surface runoff concentration and relative contributions of channel and slope erosion. J. Hydrol, 92, 59-76.

MORGAN R.P.C., 1995. Soil erosion and conservation, $2^{\text {nd }}$ ed. Longman, London.

NASH J.E., SUTCLIFFE J.V., 1970. River flow forecasting through conceptual models. Part I, a discussion of principles. J. Hydrol., 10, 282-290.

NEGEV M.A., 1967. Sediment model on digital computer. Department of Civil Engi- neering, Standford University, Standford, California, Technical Report, 76.

OLIVRY J.C., BRICQUET J.P., THIEBAUX J.P., NKAMDJOU S., 1988. Transport de matière sur les grands fleuves des régions intertropicales : les premiers résultats des mesures de flux particulaires sur le bassin du fleuve Congo. Proceedings of the Porto Allegree Symposium "Sediment Bugdet ", IAHS Publication, 174, 509-521.

OLIVRY J.C., BRICQUET J.P., BAMBA F., DIARA M., 1995a. Le régime hydrologique du Niger supérieur et le déficit des deux dernières décennies. Comptes rendus de la conférence "Grands Bassins Fluviaux ", ORSTOM, 22-24 Novembre 1993, Paris, France, 251-266.

OLIVRY J.C., GOURCY L., TOURE M., 1995b. Premiers résultats sur la mesure des flux de matières dissoutes et particulaires dans les apports du Niger au Sahel. Comptes rendus de la conférence "Grands Bassins Fluviaux ", ORSTOM, 22-24 novembre 1993, Paris, France, 281-292.

ORANGE D., 1992. Hydroclimatologie du Fouta Djalon et dynamique actuelle d'un vieux paysage latéritique. Sciences Géologiques, Mémoire, 93, Strasbourg, France, $198 \mathrm{p}$.

PICOUET C., 1999. Géodynamique d'un hydrosystème tropical peu anthropisé, le bassin supérieur du Niger et son delta intérieur. Th. Doct. Univ. Montpellier, France. $386 \mathrm{p}$.

PINHEIRO A., CAUSSADE B., 1996. Modélisation de la pollution diffuse agricole : 
cas des matières en suspension et du phosphore. Revue de l'Agence de l'Eau Adour-Garonne, 67, 12-18.

WALLING D.E., 1977. Assessing the accuracy of suspended sediment rating curves for a small basin. Water Ressour. Res., 13, 531-538.

WALLING D.E., WEBB B.W., 1981. The reliability of suspended sediment load data. Proceedings about Erosion and Sediment Transport Measurement, Florence, june 1981. IAHS Publication, 133, 177194.

WICKS J.M., BATHURST J.C., 1996. SHESED: a physically based, distributed erosion and sediment yield component for the SHE hydrological modelling system. J. Hydrol., 175, 213-238.

ZHANG J., HUANG W.W., SHI M.C., 1990. Huanghe (Yellow River) and its estuary: sediment origin, transport and deposition. J. Hydrol., 120, 203-223. 